Gut, 1979, 20, 590-595

\title{
Grey scale ultrasound in Crohn's disease
}

\author{
S. HOLT ${ }^{1}$ AND E. SAMUEL
}

From the University Department of Therapeutics and Clinical Pharmacology and Department of Medical Radiology, The Royal Infirmary, Edinburgh

SUMMARY Grey scale ultrasound examination was performed in 20 patients with Crohn's disease of varying extent and severity. In 15 of the 20 patients studied, thickening of the wall of the terminal ileum and caecum, with adjoining inflammatory changes in the mesentery, produced recognisable patterns on longitudinal and transverse ultrasonographs. Grey scale ultrasound, although not providing the specificity or detail of conventional radiology, can be helpful in the assessment of ileocaecal Crohn's disease, particularly in younger patients where the radiation hazards of repeated $x$-ray examinations are undesirable.

Grey scale ultrasound examination is a simple, noninvasive investigation that has wide application in the diagnosis of abdominal masses. To date, abdominal ultrasound has been used mainly to define abnormalities in organs other than the bowel, because the gas content of intestinal loops results in total disruption of the ultrasonic beam. An added problem is the mobility of bowel loops which tends to give rise to inconstant images on sectional ultrasonographs (Holm, 1971). However, thickening of the bowel wall is usually associated with relative immobility of bowel loops and produces a recognisable pattern on grey scale ultrasound, which on occasion may give rise to a characteristic image (Lutz and Petzoldt, 1976; Weissberg et al., 1977; Holt and Samuel, 1978).

Patients with Crohn's disease often require repeated assessment of the activity and extent of their disease within the bowel. Until recently, assessment of the extent of disease was adequately achieved only by barium contrast radiography with its drawbacks of repeated irradiation and patient discomfort. Our aim in the present study was to evaluate the potential of grey scale ultrasound in the assessment of ileocaecal Crohn's disease, thereby perhaps obviating the need for repeated abdominal $x$-rays after the initial diagnosis had been made.

\section{Methods}

SUBJECTS

Twenty patients with known regional enteritis of

1Address for correspondence: Dr S. Holt, University Department of Therapeutics and Clinical Pharmacology, The Royal Infirmary, Edinburgh EH3 9YW.

Received for publication 17 January 1979 varying severity and extent were examined by grey scale ultrasound, without knowledge of the previous clinical or radiological findings (Table 1). The patients were supine during the ultrasound examination and multiple transverse and longitudinal scans of the lower abdomen were performed at approximately $5 \mathrm{~cm}$ intervals. The scans were performed using a Diasonograph $\mathbf{4 0 0 0}$ machine with a transducer frequency of either 2.25 or $3.5 \mathrm{MHz}$ and recordings were obtained on multiformat $x$-ray film. All of the patients had Crohn's disease affecting the terminal ileum and, in six cases, clinical examination had identified palpable bowel loops in the right iliac fossa (Table 1). In each case, conventional contrast radiography of the small and large bowel, performed within one year of the ultrasound study, had confirmed the presence of Crohn's disease.

The ultrasound operator reported the presence or absence of abnormalities in the lower abdomen indicative of inflammatory involvement of the terminal ileum and caecum. After this assessment, the ultrasonographic findings were compared with the radiologically observed distribution of disease in the corresponding area of the abdomen (Table 1). Ultrasonographs obtained from patients with abdominal space-occupying lesions arising from bowel loops affected by pathology other than Crohn's disease were also examined (Table 2). The specificity of the results obtained in the Crohn's disease group were assessed by comparison with the latter group.

\section{Results}

Grey scale ultrasound was successful in defining abnormalities in $\mathbf{1 5}$ of the 20 patients studied (Table 1). In the five patients in whom ultrasound was negative, only a short segment of diseased small 
Table 1 Clinical, radiological, and ultrasound findings in 20 patients with Crohn's disease

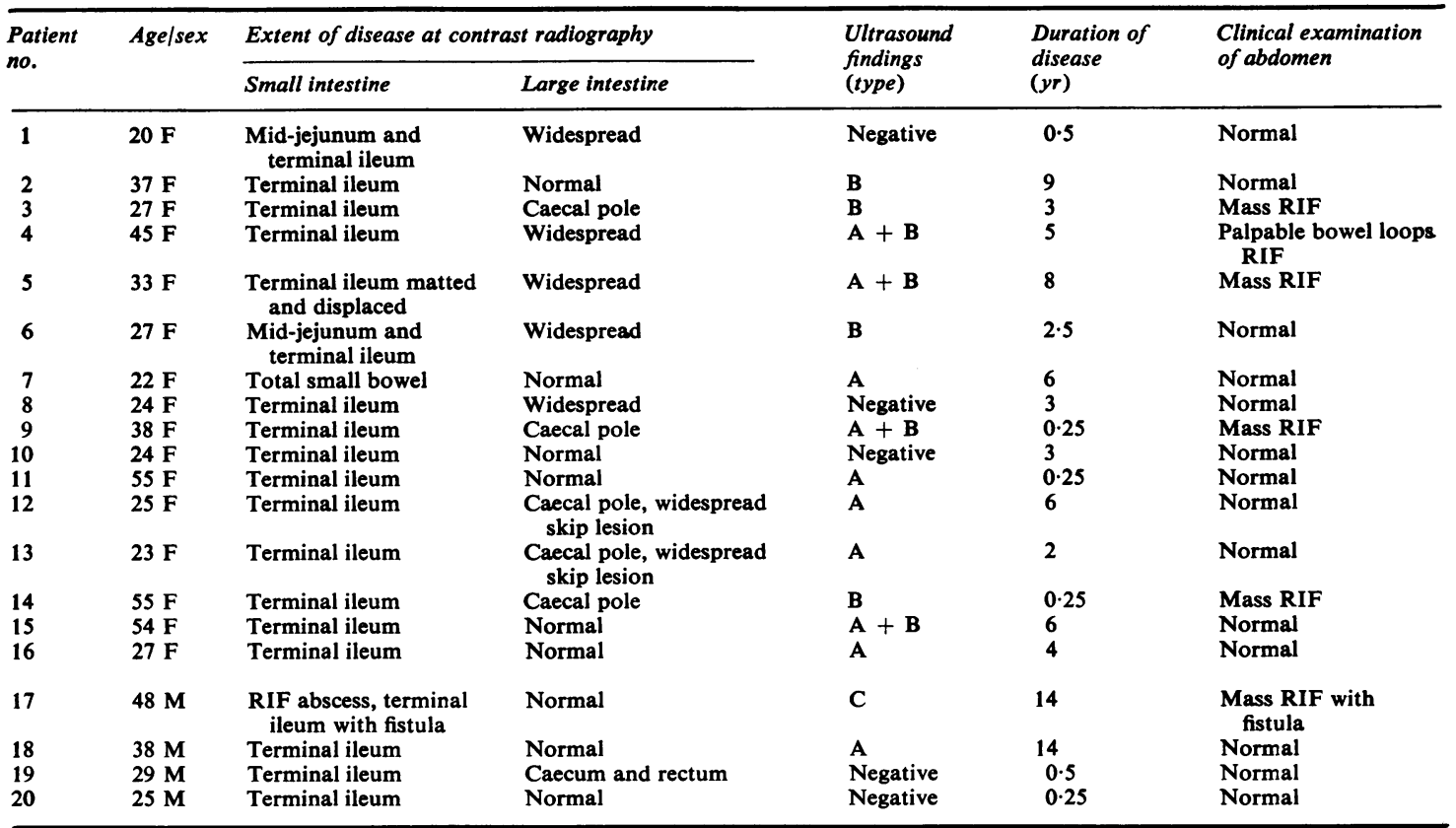

RIF : right iliac fossa. F : female. $M$ : male. Type $A, B$, and $C$ (see text).

Table 2 Clinical, radiological, and ultrasound findings in patients with diseases resulting in bowel thickening

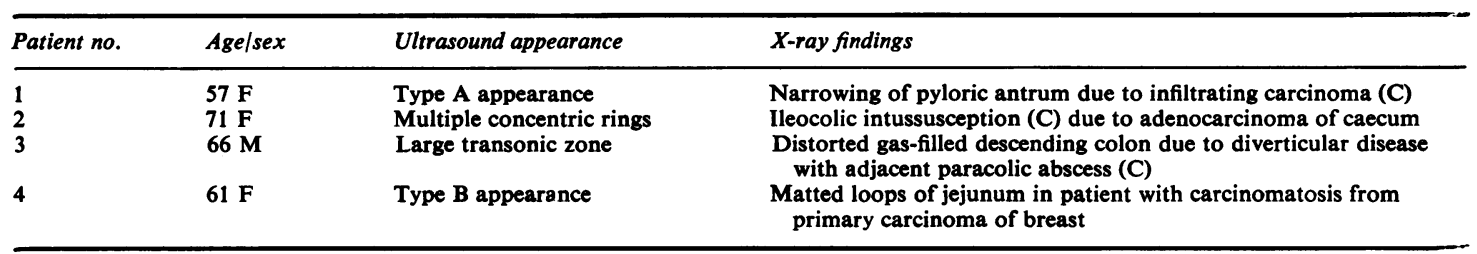

C: confirmed at operation or post mortem.

bowel had been observed by contrast radiography. In four of these five patients, their disease was in clinical remission. Three main patterns were identified on transverse and longitudinal ultrasonographs. The type A appearance was observed in 10 of the 20 patients studied. This image was represented by areas of sonodensity and sonolucency, corresponding with intestinal lumen and diffuse thickening of the bowel wall. This appearance was most often seen by transverse scanning (Fig. 1). The type B appearance was present in eight of the 20 patients. It consisted of multiple central densely reflective areas of varying size and distribution in a sonolucent zone and was considered to represent coils of bowel matted together (Fig. 2). Laparotomy in case 15 (Table 1) had confirmed the suspicion that the type $A$ and type $B$ appearance represented an inflammatory mass of bowel. The type $\mathrm{C}$ appearance (Fig. 3) was made up of a variable combination of $A$ and $B$ associated with a large transonic area, indicating a local abscess. This appearance was seen only in case 17 (Table 1). Both the type A and type B appearances were seen together in four of the 20 patients studied. In three of the four patients with the combined appearance, a palpable abdominal mass was present.

\section{Discussion}

Bowel thickening and aggregation of intestinal coils due to Crohn's disease of the terminal ileum and caecum presents a varied but recognisable grey scale echographic appearance. The most consistent image was the type A appearance. This single area of sonodensity surrounded by a ring of sonolucency corresponds with the bowel lumen and contents, and the thickened gut wall. The type A appearance is not 


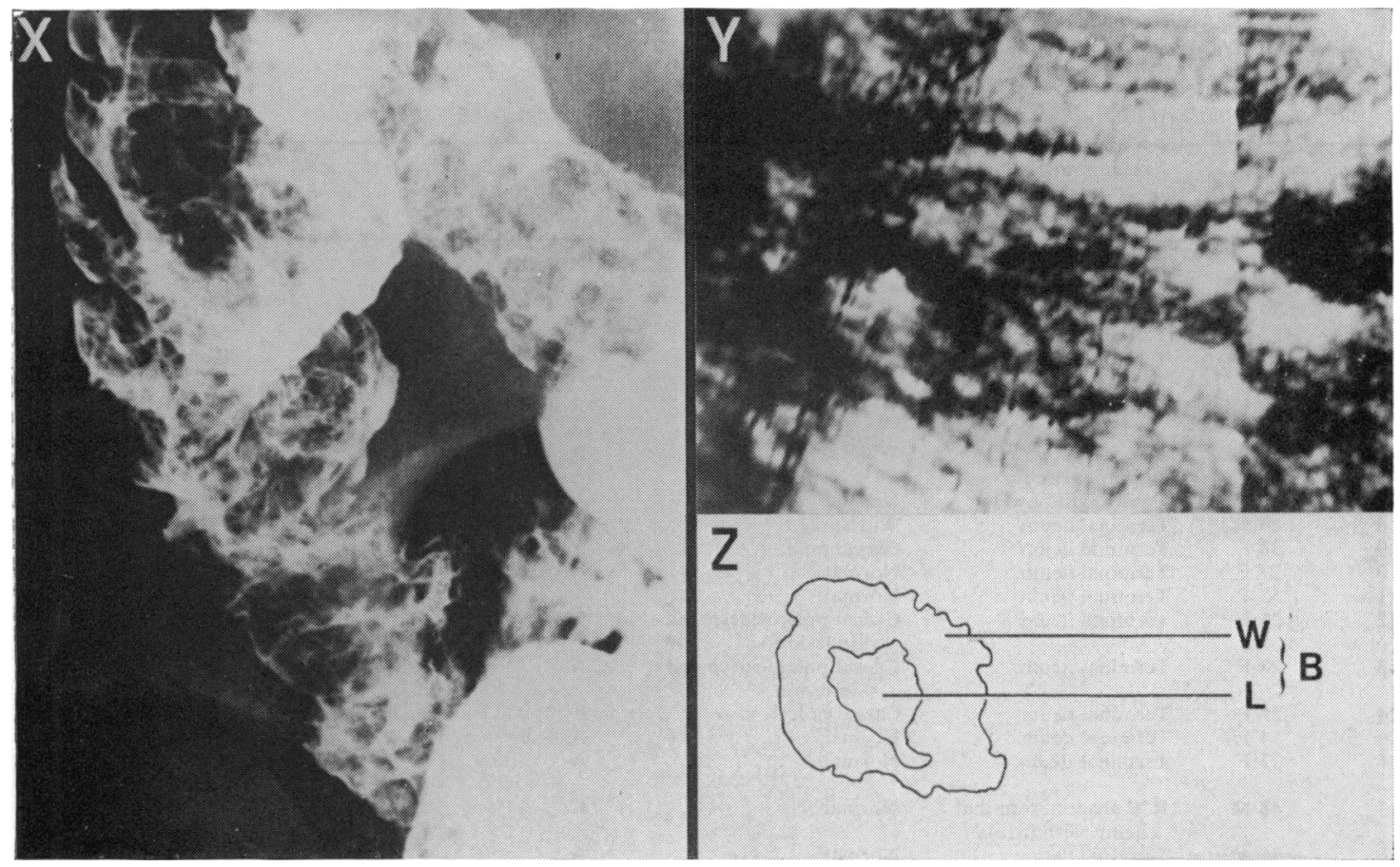

Fig. 1 The barium follow-through $(X)$ demonstrates a contracted caecal pole and narrow terminal ileum. The transverse ultrasonograph $(Y)$ shows the type A appearance. The diagrammatic representation of the ultrasonograph $(Z)$ indicates that this image $(B)$ is formed by the lumen $(L)$ and diffusely thickened wall $(W)$ of the caecum.

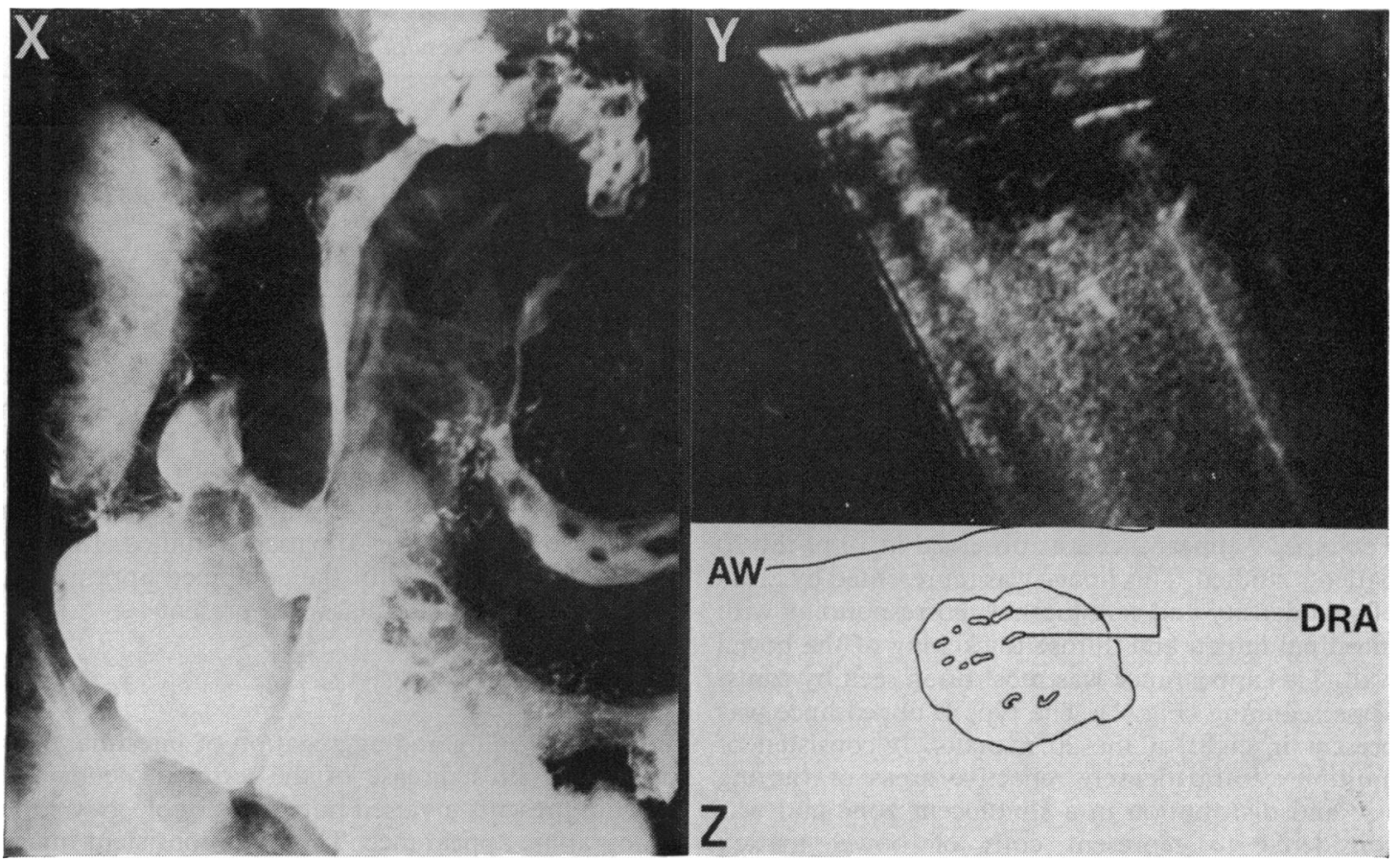

Fig. 2 The barium follow-through $(X)$ shows extensive Crohn's disease of the terminal ileum with matted displaced loops of small bowel and the longitudinal ultrasonograph $(Y)$ demonstrates the corresponding type B appearance. The diagrammatic representation of the ultrasonograph $(Z)$ indicates that this image is composed of multiple densely reflective areas $(D R A)$. AW is anterior abdominal wall. 


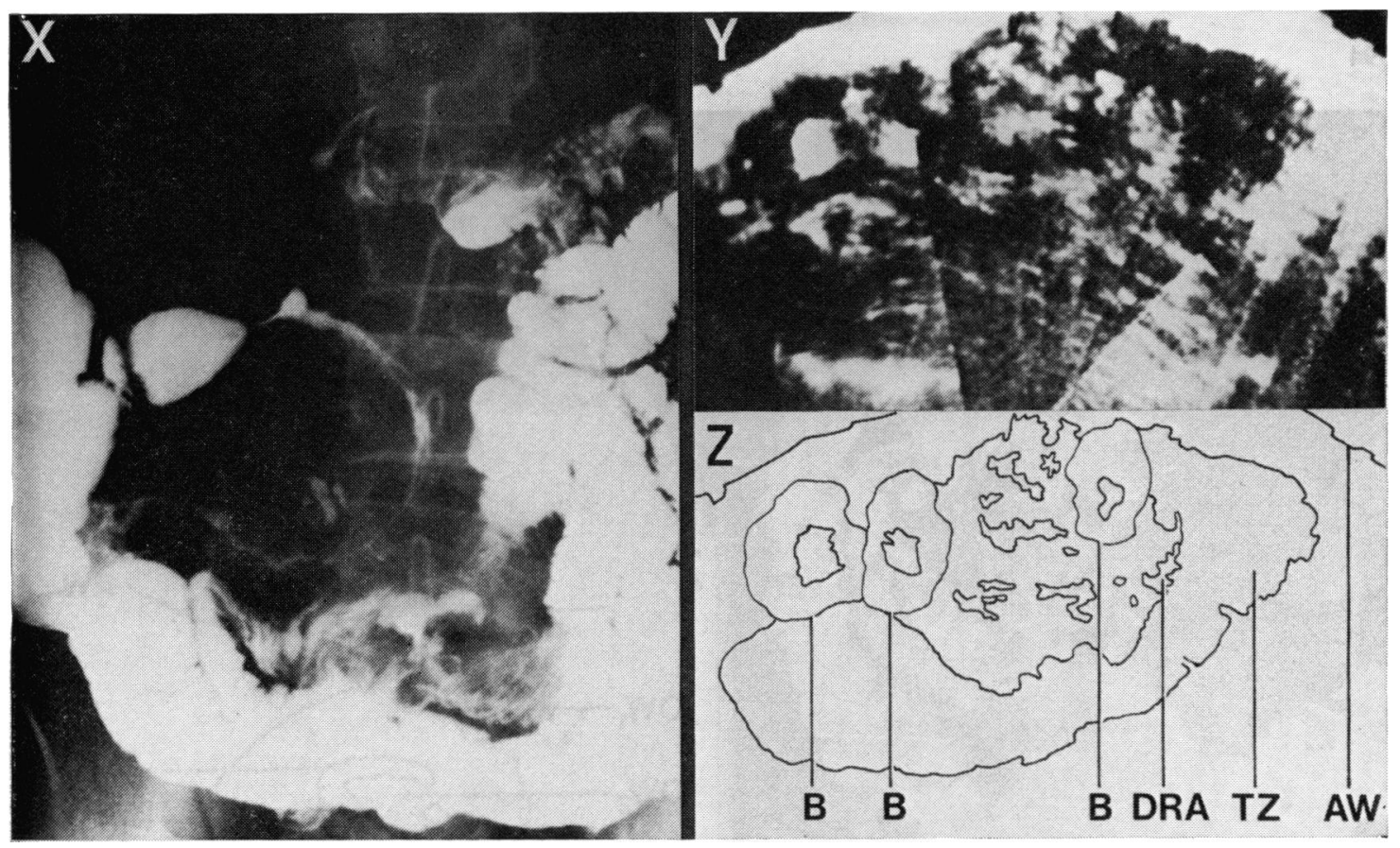

Fig. 3 The barium follow-through $(X)$ shows a stretched loop of ileum around an abscess cavity. The transverse uitrasonograph $(Y)$ demonstrates the type $C$ appearance which is indicated in the diagram $(Z)$ to be composed of type $A$ images $(B)$, densely reflective areas $(D R A)$, and an adjacent transonic zone $T Z$. AW is anterior abdominal wall.

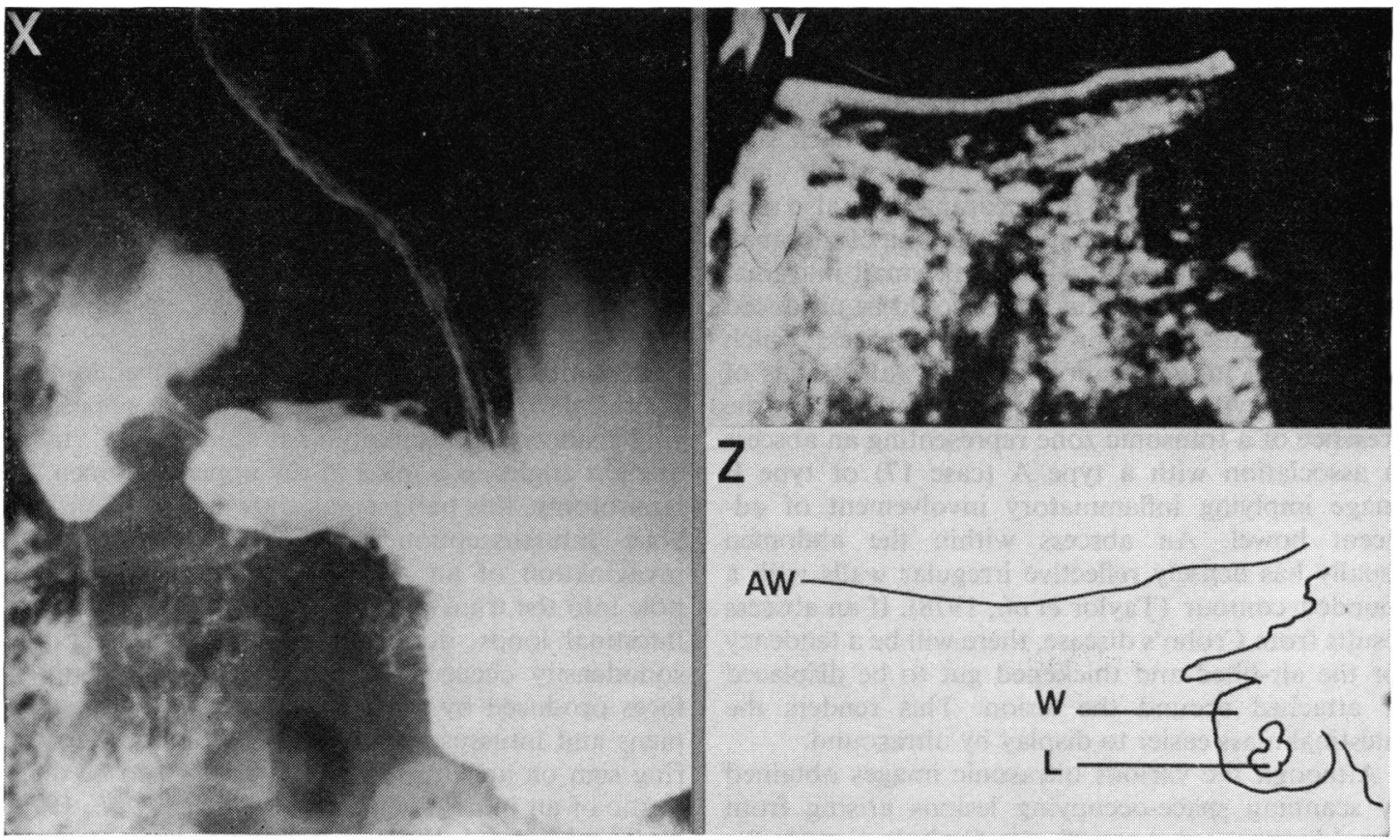

Fig. 4 The barium meal $(X)$ demonstrates narrowing of the pyloric antrum by infiltrating carcinoma. The longitudinal ultrasonograph $(Y)$ shows a type A image which is shown diagrammatically $(Z)$ to be formed by the lumen $(L)$ and diffusely thickened wall $(W)$ of the pyloric antrum. AW is anterior abdominal wall. 

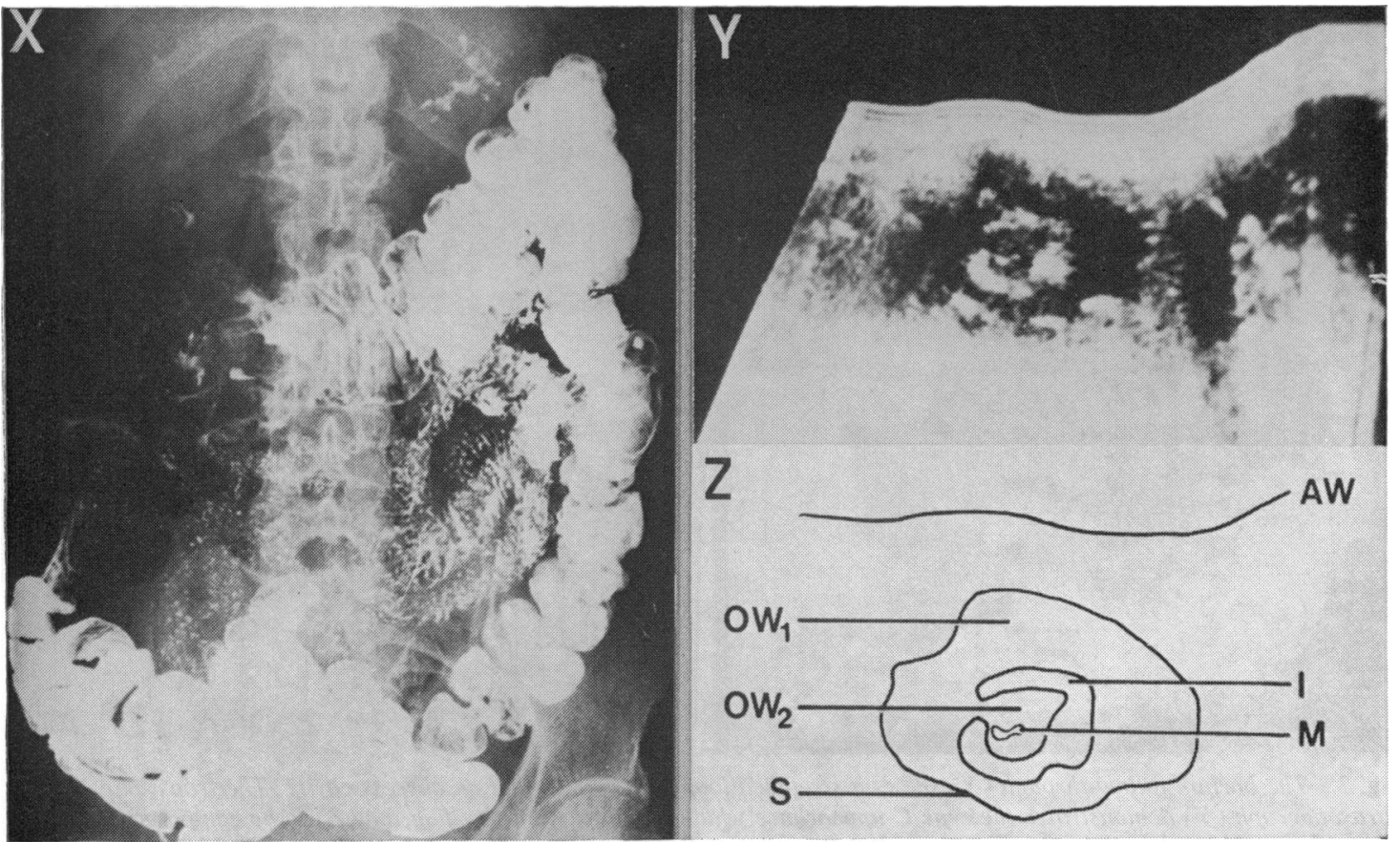

Fig. 5 The barium follow-through $(X)$ shows an ileocolic intussusception and the longitudinal scan $(Y)$ demonstrates the 'multiple concentric ring sign' of an intussusception. The diagram $(Z)$ of the ultrasonograph indicates the formation of this image by the oedematous wall of the intussusceptum $\left(\mathrm{OW}_{1}\right)$, the oedematous wall of the intussuscipiens $\left(\mathrm{OW}_{2}\right)$, the interface $(I)$, and the serosa $(S) . A W$ is anterior abdominal wall.

specific for Crohn's disease and has been noted in relationship with carcinoma of the stomach and intestines (Lutz and Petzoldt, 1976; Holt and Samuel, 1978). The type B appearance was also seen in association with metastatic carcinoma of the small bowel, producing a matted mass of small intestinal loops (Table 2). This latter image could be produced by any inflammatory or malignant process which would result in aggregation of thick-walled coils of bowel. The type $\mathrm{C}$ appearance is characterised by the presence of a transonic zone representing an abscess in association with a type A (case 17) or type B image implying inflammatory involvement of adjacent bowel. An abscess within the abdomen usually has densely reflective irregular walls with a rounded contour (Taylor et al., 1978). If an abscess results from Crohn's disease, there will be a tendency for the air-filled and thickened gut to be displaced or attached around the lesion. This renders the intestinal mass easier to display by ultrasound.

Although the various ultrasonic images obtained by scanning space-occupying lesions arising from bowel loops are not specific for Crohn's disease, the anatomical location may often allow identification of the section of gut from which the mass has arisen.
The type A appearance that indicates thickened and stenosed bowel wall was seen in case 1 (Table 2) and resulted from an infiltrating carcinoma of the pyloric antrum (Fig. 4). In this patient, the image was obtained by longitudinal scanning of the upper quadrant of the abdomen to the left of the mid-line suggesting an origin within stomach or transverse colon.

In another patient (case 2, Table 2) abdominal grey scale ultrasound revealed multiple concentric ring shadows on a longitudinal scan (Fig. 5), taken at right angles to a mass in the upper abdomen. At laparotomy, this patient was shown to have an ileocolic intussusception which had resulted from invagination of an adenocarcinoma of the caecal pole into the transverse colon. With invagination of intestinal loops, double areas of sonolucency and sonodensity occur that correspond with the interfaces produced by abutting walls of the intussuscipiens and intussusceptum. This multiple concentric ring sign on an ultrasonograph appears to be diagnostic of an intussusception (Weissberg et al., 1977; Holt and Samuel, 1978). A paracolic abscess (case 3, Table 2) arising from any cause may give rise to a transonic zone but this image was encountered in the 
left lower quadrant of the abdomen and was not accompanied by adjacent thickened bowel, so that there was no associated type A or type B appearance.

Ultrasonic investigation is a rapid, safe, noninvasive technique which does not require any special preparation of the patient. Any method which would reduce the repeated radiation exposure that patients with Crohn's disease require, is advantageous. Furthermore, barium studies visualise only the lumen of the terminal ileum or caecum and the dimensions of the bowel thickening or matting cannot be assessed. With ultrasound, it may be possible in a large proportion of cases to demonstrate the actual size of the involved area of bowel, as well as local abscess formation. It may also have a use in the identification of bowel thickening in pregnant patients and may be used in individuals with intestinal obstruction, in whom contrast radiography is contraindicated.

In five of the 20 patients examined by ultrasound, gas-filled bowel produced degradation of the ultrasonic beam, which made it impossible to detect the intestinal lesion. However, useful information obtained in $75 \%$ of the patients with Crohn's disease examined by ultrasound illustrates the potential of this technique in the diagnosis of lesions arising from intestinal loops. With periodic examination, ultrasound may provide a way of assessing advanced regional complications in Crohn's disease by permitting the recognition of thickening of the bowel, matted loops of bowel, and abscess cavities. Although ultrasound cannot replace contrast radiography either in the diagnosis or in the detection of early and localised lesions in Crohn's disease, it is a simple test with a high degree of patient acceptability that can be used repeatedly as an adjunct to the evaluation of ileocaecal Crohn's disease.

We thank the physicians and surgeons of The Royal Infirmary, Edinburgh, for permission to study their patients and in particular Dr R. C. Heading who also provided constructive criticism. The Editor of Gastrointestinal Radiology kindly gave permission for the reproduction of Figs 4 and 5.

\section{References}

Holm, H. H. (1971). Ultrasonic scanning in the diagnosis of space-occupying lesions of the upper abdomen. British Journal of Radiology, 44, 24-36.

Holt, S., and Samuel, E. (1978). The multiple concentric ring sign in the ultrasonographic diagnosis of intussusception. Gastrointestinal Radiology, 3, 307-309.

Lutz, H. T., and Petzoldt, R. (1976). Ultrasonic patterns of space-occupying lesions of the stomach and the intestine. Ultrasound Medical Biology, 2, 129-132.

Taylor, K. J. W., Wasson, J., De Graaff, C., Rosenfield, A. T., and Andriole, V. T. (1978). Accuracy of grey scale ultrasound diagnosis of abdominal and pelvic abscesses in 220 patients. Lancet, 1, 83-84.

Weissberg, D. L., Scheible, W., and Leopold, G. R. (1977). Ultrasonic appearance of adult intussusception. Radiology, 124, 791-792. 